\title{
The Quality of Primary Care Experienced by Health Center Patients
}

\author{
Leiyu Shi, DrPH, MBA, MPA, Lydie A. Lebrun-Harris, PhD, MPH, \\ Sarika Rane Parasuraman, PhD, MPH, Jinsheng Zhu, MEc, and \\ Quyen Ngo-Metzger, MD, MPH
}

Background: We investigated the quality of the primary care experienced by health center (HC) patients and investigated whether race/ethnicity and insurance coverage were significantly associated with patients' experiences.

Methods: Cross-sectional data came from the 2009 Health Center Patient Survey. Outcomes included 10 measures of patients' experiences with primary care domains, including: (1) accessibility, (2) communication, (3) comprehensiveness, and (4) coordination of care.

Results: Patients who received care at HCs reported high-quality primary care, particularly regarding accessibility and communication. For example, more than $94 \%$ of patients reported that their HC location was convenient, and more than $94 \%$ reported that their provider adequately explained what they wanted to know. After adjusting for sociodemographic characteristics, few significant racial/ethnic and insurance-related disparities were observed. In the domains of comprehensiveness and coordination, insured patients generally had better experiences than uninsured patients. For instance, Medicaid-insured patients had higher odds of reporting that HC staff helped them arrange medical appointments at other health care settings than uninsured patients (odds ratio, 2.04; 95\% confidence interval, 1.353.09).

Conclusions: As safety-net providers for vulnerable populations, HCs provide high-quality primary care and do not exhibit the extent of disparities that exist in other US health care settings. Continued efforts are necessary to address insurance-related disparities, particularly among uninsured patients. (J Am Board Fam Med 2013;26:768-777.)

Keywords: Ethnicity, Health Care Disparities, Health Insurance, Quality of Health Care, Race

The Institute of Medicine defines quality of care as "the degree to which health services increase the

\footnotetext{
This article was externally peer reviewed.

Submitted 11 February 2013; revised 24 April 2013; accepted 15 May 2013.

From the Department of Health Policy and Management, Johns Hopkins Bloomberg School of Public Health, Baltimore, MD (LS); Johns Hopkins Primary Care Policy Center, Baltimore, MD (LS; SRP, JZ); the Office of Quality and Data, Bureau of Primary Health Care, Health Resources and Services Administration, U.S. Department of Health and Human Services, Rockville, MD (LAL-H, QN-M).

Current affiliation: Office of Planning, Analysis and Evaluation, Health Resources and Services Administration (LAL-H), and the U.S. Preventive Services Task Force Program, Agency for Healthcare Research and Quality (QNM), U.S. Department of Health and Human Services, Rockville, $\mathrm{MD}$.

Funding: The authors acknowledge the financial support for this study provided by the Health Resources and Services Administration (HRSA) of the U.S. Department of Health and Human Services (HHS) (Contract No. HHSH250200646022I).
}

likelihood of desired health outcomes and are consistent with current professional knowledge." Quality of care may be further categorized into technical aspects and interpersonal aspects. Technical aspects of quality are those that capture the application of technology, medical tools, or delivery of care protocols by providers, whereas interpersonal aspects of quality are those describing interactions between a patient and either providers or

Conflict of interest: none declared.

Disclaimer: The views expressed in this publication are solely the opinions of the authors and do not necessarily reflect the official policies of HHS, HRSA, or the Agency for Healthcare Research and Quality, nor does mention of the department or agency names imply endorsement by the U.S. government.

Corresponding author: Leiyu Shi, DrPH, MBA, MPA, Johns Hopkins Primary Care Policy Center, Department of Health Policy and Management, Johns Hopkins Bloomberg School of Public Health, 624 North Broadway, Hampton House Room 409, Baltimore, MD 21205 (E-mail: Ishi@jhsph.edu). 
the health care system. Recent research has emphasized the importance of measuring patients' selfreports and ratings of their health care experiences. Patient-centered quality measurement tools have been developed to capture these perspectives, the most notable of which is the Consumer Assessment of Health care Providers and Systems collection of surveys. ${ }^{2,3}$ Studies have shown that positive patient experiences with the care and services they receive from providers and within health care settings are associated with several positive outcomes, including increased health care utilization, heightened compliance with prescribed treatment regimens, and fewer missed appointments. ${ }^{4,5}$

Primary care plays a critical role in the health and well-being of patients, setting a framework for health maintenance and early detection and treatment of disease. ${ }^{6,7}$ In its landmark report, the Institute of Medicine listed several defining attributes of primary care, including accessibility, coordination, and comprehensiveness. ${ }^{8}$ Accessibility refers to the degree to which a patient is able to access and use health services when a health problem must be addressed. Coordination describes care and services that operate seamlessly along a continuum through the health care system. Comprehensiveness refers to the role of primary care within the larger health system and indicates the ability of primary care settings to assist patients with related services. More recent efforts also highlight the significance of provider-patient communication in promoting high-quality, patient-centered care. ${ }^{9}$

The association between primary care, access to care, and health outcomes also has been well documented in the literature. ${ }^{10,11}$ In particular, individuals who experience a lack of access to primary care are more likely to sustain serious morbidity from preventable conditions because of missed screening services (eg, hypertension, stroke) and to suffer from severe health or chronic conditions requiring hospitalizations or emergency care. ${ }^{12,13}$ Health insurance has similarly been shown to be strongly associated with access to care. ${ }^{14}$ Despite this knowledge, significant differences in access to quality primary care exist across groups. Being poor or belonging to a racial/ethnic minority is generally associated with having unmet health care needs, inferior access to primary care, and worse health outcomes. ${ }^{15,16}$ Furthermore, those who are poor or lack health insurance are more likely to suffer higher rates of morbidity and mortality. ${ }^{16}$ These disparities bring enormous economic and social consequences for the United States; thus, reducing or eliminating disparities in health and health care is a priority for the nation. ${ }^{1,16}$

Federally funded health centers (HCs) play an important role in addressing health disparities in the United States. Originating in the 1960s, HCs aim to provide comprehensive primary care and support services to people living in medically underserved areas. ${ }^{17}$ HCs offer a wide range of services, including primary care services, mental/behavioral health care, dental care, and supportive services (eg, transportation, translation services, health education). ${ }^{18,19}$ As of 2011, 1128 HCs with more than 8000 sites served more than 20 million patients across the nation. HCs typically provide care to underserved communities comprising patients who are predominantly poor, uninsured/underinsured, or racial/ethnic minorities. More than $90 \%$ of patients who seek care from HCs have incomes below $200 \%$ of the federal poverty level; more than $60 \%$ are racial/ethnic minorities, and nearly $40 \%$ are uninsured. ${ }^{19}$

It is critical to evaluate services in primary care settings that provide care for underserved or vulnerable populations, such as HCs, as well as to examine factors that exist in the relationship between primary care and health outcomes, to accomplish the nation's goal of reducing or eliminating disparities in health and health care. However, there have been few studies in HC settings investigating differences in the quality of the primary care experienced by patients with different insurance statuses or those of different racial/ethnic groups. The purpose of this study was to investigate the quality of the primary care experienced by patients attending an HC. In particular, this study (1) described the quality of the primary care experience from the perspective of patients attending an $\mathrm{HC},(2)$ examined differences in the primary care experience among patients of different racial/ethnic groups and insurance coverages, and (3) investigated whether race/ethnicity or insurance coverage were significantly associated with patients' primary care experience after accounting for other covariates.

\section{Methods}

\section{Data Source}

This study employed a cross-sectional analysis using data from the 2009 Health Center Patient Sur- 
Table 1. Dependent Measures of Accessibility, Communication, Comprehensiveness, and Coordination

\begin{tabular}{lll}
\hline Category & \multicolumn{1}{c}{ Measure } & Variable \\
\hline Accessibility & Have a usual source of care & Yes/more than one place vs. no \\
& Convenience of HC location & Excellent/very good/good vs. fair/poor \\
& Ability to be seen at HC & Excellent/very good/good vs. fair/poor \\
Communication & Provider listens to patient & Excellent/very good/good vs. fair/poor \\
& Provider explains what patient wants to know & Excellent/very good/good vs. fair/poor \\
& Provider gives good advice and treatment & Excellent/very good/good vs. fair/poor \\
Comprehensiveness & Staff assisted patient with application for government & Yes vs. no \\
& benefits & \\
& Staff helped patient obtain transportation to medical & Yes vs. no \\
& appointments & Yes vs. no \\
Cooff helped patient obtain free medication & Yes vs. no \\
& Staff helped patient arrange medical appointments at & \\
\hline
\end{tabular}

HC, health center.

vey, a nationally representative survey sponsored by the Health Resources and Services Administration. The 2009 Health Center Patient Survey has a probability sample of 4562 patients representing more than 16 million patients seen at HCs during 2009.

A complex, 3-stage sampling scheme was employed to ensure that the final set of survey responses was a nationally representative sample. First, eligible HCs were randomly selected from a larger sampling frame, and then eligible sites were selected from within each HC. Finally, eligible patients were selected from each $\mathrm{HC}$ site. Eligible patients were those who had at least one medical visit to an eligible $\mathrm{HC}$ site in the past 12 months. First-stage sampling was stratified by funding stream, HC size, US Census region, urban/rural location, and number of service sites per HC. The second stage selected up to 3 sites per HC. The third stage selected individual patients within service sites, and interviews were completed between September and December 2009. Computer-assisted personal interviews were conducted in English and Spanish and lasted about 50 minutes. After completing the interview, respondents received $\$ 25$ in cash or as a gift card. Institutional review board approval was obtained from Research Triangle International, the organization in charge of data collection. Local institutional review board or other committee approvals were obtained where necessary.

Overall, 188 grantees were sampled with probability proportional to HC patient volume (91\% response rate at the grantee level). The second stage selected up to 3 sites per grantee. Data were collected from a total of 432 sites (97\% response rate at the site level). The third stage selected individual patients within service sites, and a consecutive sample was selected from patients who entered the site and consented to participate in the survey. Among 8275 patients initially invited to participate, 5965 (72\%) consented to participate. Of these, $1323(16 \%)$ were ineligible because they did not have a visit during the past year, and another $80(1.0 \%)$ did not complete the interviews. A total of 4562 patients completed interviews. Thus, the response rate was $55 \%$ among patients initially identified, and the response rate was $98 \%$ among patients confirmed to be eligible.

\section{Measures}

The 10 outcome measures used in this study describe distinguishing attributes of primary care that capture the quality of patients' primary care experiences in the HC. All measures were coded as dichotomous variables. Patient-reported dependent measures included 3 measures of accessibility, 3 measures rating communication, 3 measures reporting comprehensiveness, and 1 measure reporting coordination. These measures are described in Table 1.

The comprehensiveness measures in particular are unique to HC settings; most private practices would not define comprehensiveness in the same way and would not typically provide these types of services. However, we included the measures here because they comprise types of "enabling services" 
that are considered critical to ensuring access to quality services for the HC patient population.

The main independent variables were race/ethnicity and insurance status. Race/ethnicity was categorized into 5 groups: Hispanic, non-Hispanic white, non-Hispanic black, non-Hispanic Asian, and other. Insurance status was categorized into 5 groups: uninsured, Medicaid, Medicare, private insurance, and other public insurance, including, for example, military or other state-sponsored plans. Several additional covariates were included in the analysis: age, sex, marital status, employment status, education, language (ie, English fluency), and perceived health status.

\section{Analysis}

Descriptive statistics were first obtained for all patients attending an HC. Next, bivariate analyses were performed, comparing indicators of quality of primary care across racial/ethnic and insurance categories. Finally, multivariate logistic regressions were conducted to investigate the relationship between race/ethnicity and insurance coverage and quality of the primary care experience while controlling for the potential confounding effects of demographic and socioeconomic characteristics of the HC study sample. All statistical analyses accounted for the complex sampling design used in this study by incorporating weights as well as variables identifying strata. SAS statistical software version 9.1 (SAS, Inc., Cary, NC) was used to perform all statistical analyses in this study, and 2-tailed $P$ values $\leq .05$ were considered to be statistically significant.

\section{Results}

Sociodemographic Characteristics of Patients at HCs

Table 2 shows the distribution of sociodemographic characteristics among the overall sample of patients at HCs. Weighted frequencies showed a greater proportion of female than male patients (59\% female vs. $41 \%$ male). Non-Hispanic white respondents comprised the highest proportion of any racial/ethnic group, followed by Hispanic and non-Hispanic black respondents $38 \%$ non-Hispanic white, 32\% Hispanic, 22\% non-Hispanic black). Regarding insurance coverage, most patients at HCs were either uninsured or insured by Medicaid (37\% uninsured, 33\% Medicaid). About $10 \%$ of patients had Medicare, and another $10 \%$ had private insurance. Other public insurance plans collectively accounted for the final $10 \%$ of patients.
Table 2. Health Center Patient Characteristics

$(\mathrm{n}=4562)$

Characteristics

\begin{tabular}{lc} 
Mean age, years (SE) & $34.15(1.04)$ \\
Sex & \\
Male & $1674(40.56)$ \\
Female & $2888(59.44)$ \\
Race/ethnicity & \\
Hispanic & $1976(31.99)$ \\
Non-Hispanic white & $1137(37.79)$ \\
Non-Hispanic black & $1114(21.53)$ \\
Asian/other non-Hispanic & $335(8.70)$ \\
Insurance & \\
Uninsured & $1751(36.55)$ \\
Medicaid & $1410(32.53)$ \\
Medicare & $489(9.96)$ \\
Private & $338(9.98)$ \\
Other public & $502(10.99)$ \\
Marital status & \\
Married & $1094(28.91)$ \\
Not married & $2920(71.09)$ \\
Employment & \\
Employed & $1252(37.81)$ \\
Unemployed & $2728(62.19)$ \\
Education & \\
Less than high school & \\
High school or more & $2240(46.96)$ \\
English fluency & $2023(53.04)$ \\
Language other than English & \\
English & \\
Inapplicable & \\
Perceived health status & \\
Excellent/very good/good & \\
Fair/poor & \\
\hline & \\
\hline
\end{tabular}

Data are counts (weighted percentages) unless otherwise indicated. Data are taken from the 2009 Health Center Patient Survey. Numbers may not add up to $100 \%$ because of missing data for certain variables.

SE, standard error.

Although more than half of patients reported English fluency, one fifth self-reported as not speaking English (58\% English fluency vs. 17\% no English fluency). Most patients at HCs felt they were in good health $(68 \%$ excellent/very good/good perceived health status vs. $32 \%$ fair/poor perceived health status).

\section{Race/Ethnicity and Quality of Care Experienced by Patients at HCs}

Table 3 compares quality of primary care experienced by patients at HCs across the major racial/ 


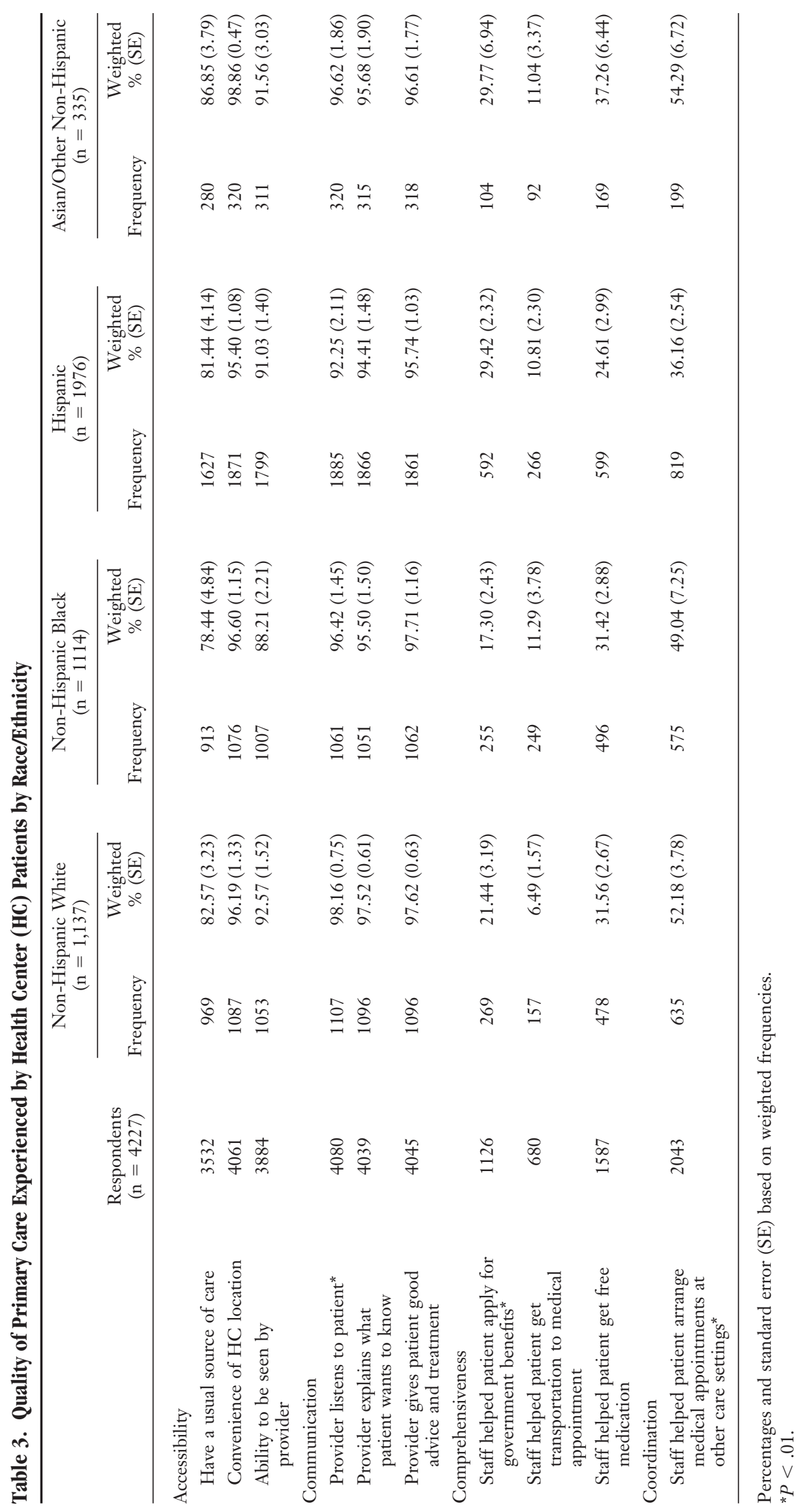


ethnic groups. With regard to accessibility indicators, more than $95 \%$ of respondents from each racial/ethnic group reported convenient $\mathrm{HC}$ location. Furthermore, there were no statistically significant racial/ethnic differences in any of the 3 accessibility measures. Overall, patients positively rated their experiences with patient-provider communication, with more than $90 \%$ of respondents in each racial/ethnic group reporting that their providers gave adequate explanations and good advice and treatment. Slightly lower proportions of Hispanic patients reported that their provider listened to them (92\%) compared with non-Hispanic white patients (98\%), non-Hispanic Asian/other patients (97\%), and non-Hispanic black patients (96\%) $(P<.01)$. Few racial/ethnic differences were observed with regard to the comprehensiveness of primary care offered at HCs, although a smaller proportion of non-Hispanic black patients reported that HC staff helped them apply for government benefits (17\%) than non-Hispanic Asian/other patients (30\%), Hispanic patients $(29 \%)$ or non-Hispanic white patients $(21 \%)(P<.01)$. In addition, racial/ethnic differences were observed with regard to coordination of care: a smaller proportion of Hispanic patients reported that HC staff helped them arrange medical appointments at other care settings (36\%) compared with non-Hispanic Asian/ other (54\%), non-Hispanic white (52\%), and nonHispanic black patients $(49 \%)(P<.01)$.

\section{Insurance Status and Quality of Care Experienced by Patients at HCs}

Table 4 compares the quality of primary care experienced by patients at HCs across the major insurance groups. No notable insurance-based differences were found with regard to communication. However, with regard to accessibility of care, a smaller proportion of uninsured patients and patients with other public insurance reported having a usual source of care (77\% for both) compared with patients insured by Medicare $(89 \%)$ or Medicaid $(87 \%)$ or privately insured patients $(86 \%)(P<$ $.05)$. In addition, virtually all privately insured patients and $98 \%$ of Medicaid patients reported convenient $\mathrm{HC}$ location, a value slightly higher than uninsured patients, patients with other public insurance (95\% for both), and patients insured by Medicare $(94 \%)(P<.05)$. Notable differences also were observed with regard to the comprehensiveness of primary care provided at HCs. A higher proportion of patients insured by Medicaid and Medicare reported that HC staff helped them apply for government benefits (32\% and 30\%, respectively) compared with uninsured or privately insured patients (18\% and 16\%, respectively), with patients with other public insurance falling in between $(P<.001)$. Regarding primary care coordination, a larger proportion of patients insured by Medicare reported that HC staff helped them arrange medical appointments at other care settings (68\%), followed by privately insured patients and patients with other public insurance $(52 \%$ for both), patients insured by Medicaid (48\%), and uninsured patients $(37 \%)(P<.001)$.

\section{Logistic Regressions: Predictors of Primary Care Experience by Patients at HCs}

Table 5 displays the results of multivariate logistic regressions performed to examine predictors of the quality of primary care experienced by patients at HCs. The analysis primarily focused on race/ethnicity and insurance status while adjusting for several sociodemographic and health status characteristics, including age, sex, marital status, employment status, educational level, English fluency, and perceived health status.

After controlling for these covariates, few racial/ ethnic disparities were observed with regard to the quality of primary care among patients at HCs. Non-Hispanic black patients had lower odds of reporting the ability to be seen by a provider (odds ratio $[\mathrm{OR}], 0.52 ; 95 \%$ confidence interval $[\mathrm{CI}]$, $0.31-0.87$ ), and Hispanic patients had lower odds of reporting that staff helped them arrange appointments elsewhere (OR, 0.55; 95\% CI, 0.340.89 ), compared with non-Hispanic white patients. On the other hand, non-Hispanic Asian/other patients had higher odds of reporting convenient HC location (OR, 5.10; 95\% CI, 1.39-18.74) and higher odds of reporting that staff helped them arrange appointments elsewhere (OR, 1.65; 95\% CI, 1.02-2.67) compared with non-Hispanic white patients.

After controlling for sociodemographic and health status covariates, several significant insurance-related disparities were observed for the quality of primary care experience among patients at HCs in terms of comprehensiveness and coordination of care. Patients insured under Medicaid and Medicare had higher odds of reporting that HC staff helped them apply for government benefits 


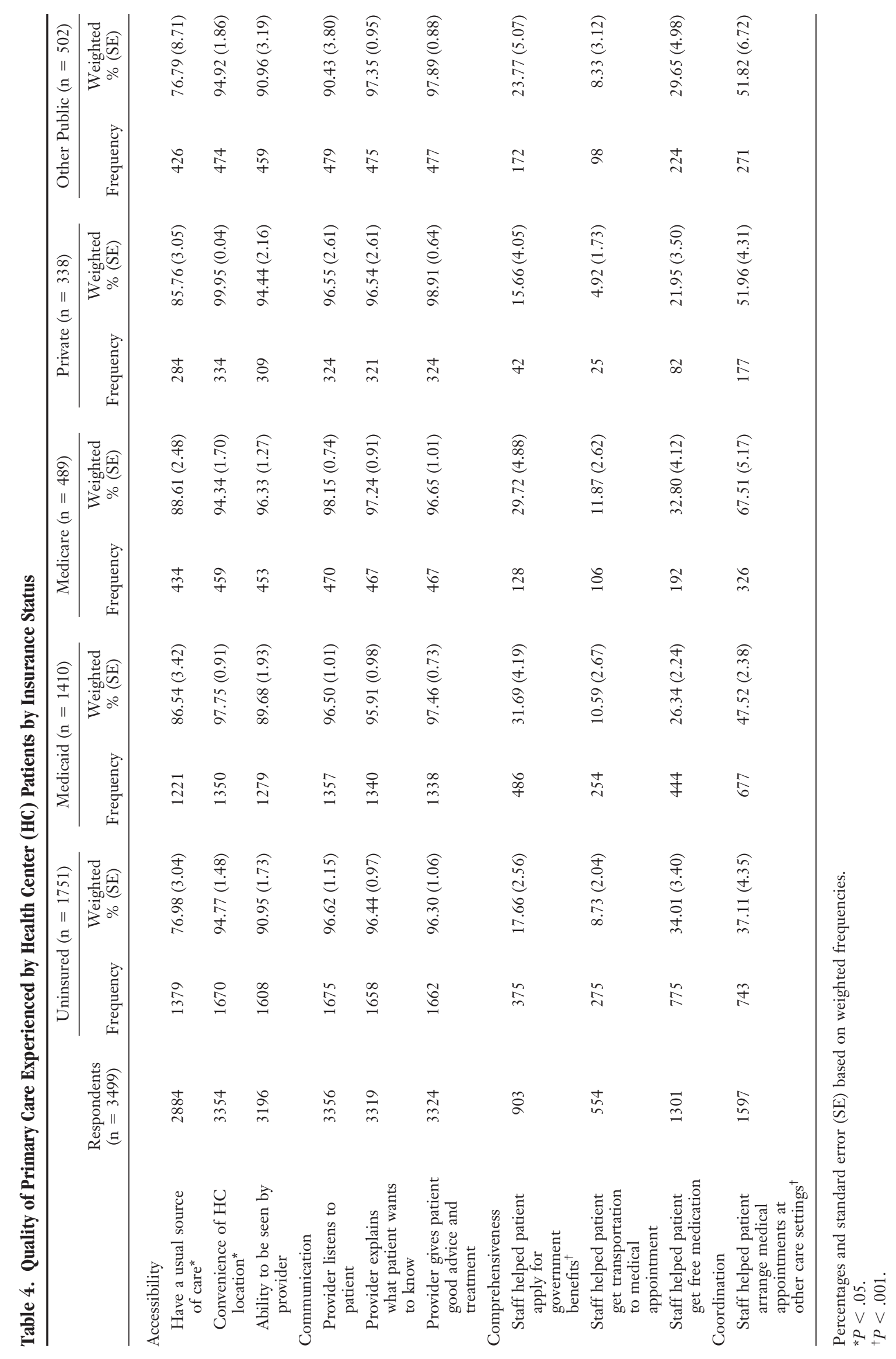



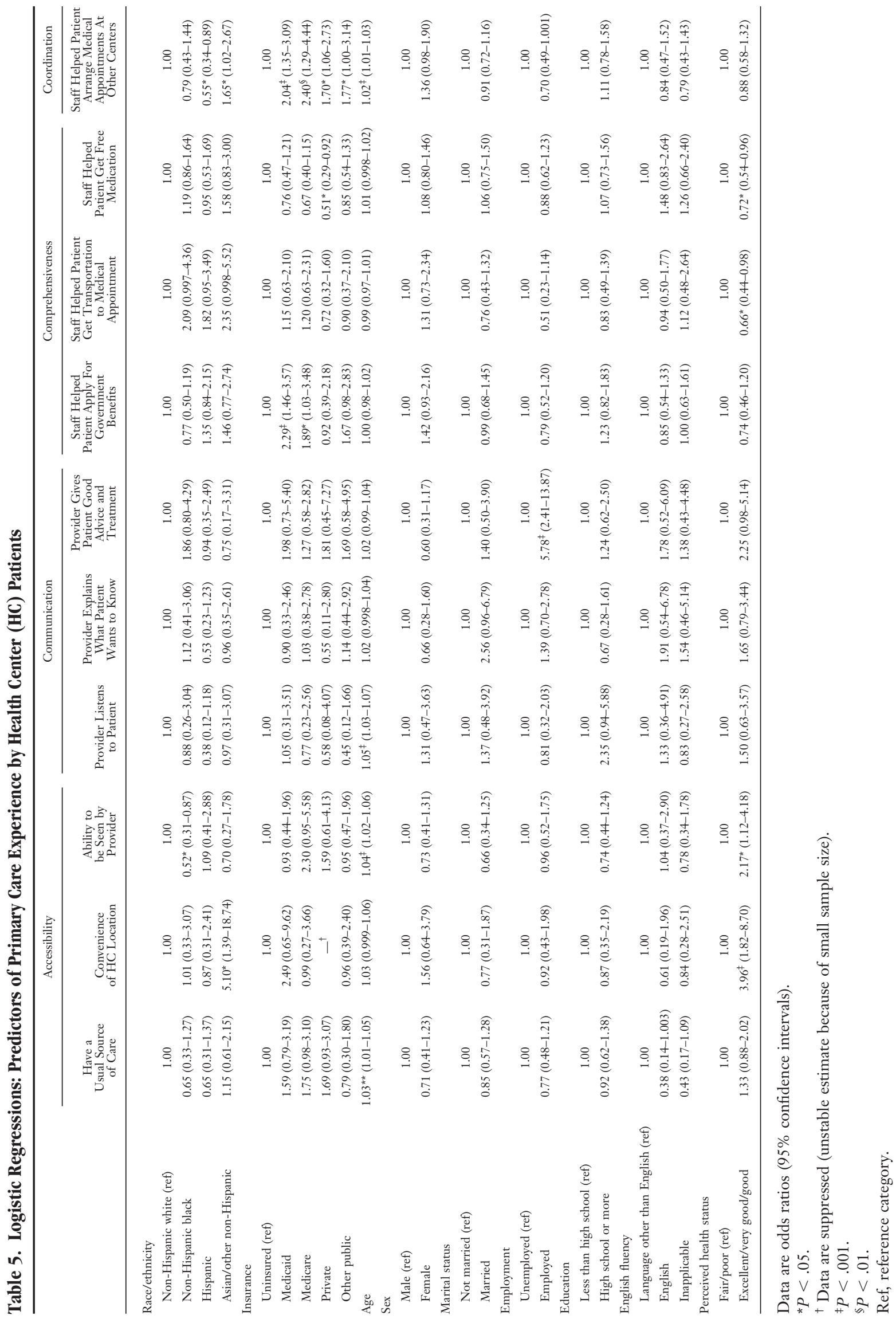
(OR, 2.29; 95\% CI, 1.46-3.57 and OR, 1.89; 95\% CI, 1.03-3.48, respectively) compared with uninsured patients. Privately insured patients had lower odds of reporting that staff helped them get free medication (OR, 0.51; 95\% CI, 0.29-0.92). Finally, compared with uninsured patients, all other insurance groups had higher odds of reporting that staff helped them arrange appointments at other care settings.

\section{Discussion}

This study is one of the first to use patient survey data to examine the quality of care experienced by a nationally representative sample of patients at HCs. In general, patients at HCs reported highquality primary care experiences, in particular with regard to measures capturing accessibility and communication. In other words, most patients at HCs were satisfied with their ability to access HC providers and services when needed and with the provider-patient communication experienced during health care visits.

Few racial/ethnic and insurance-related disparities were observed among patients at HCs, indicating that HCs provide high-quality primary care and do not exhibit the disparities that are so prevalent in other health care settings across the United States. However, uninsured patients reported less favorable primary care experiences than either privately or publicly insured patients, in particular in the domains of comprehensiveness and coordination. These findings suggest that continued efforts are necessary before health care disparities can be eliminated in HC settings. For example, additional outreach and assistance from HC staff in applying for government benefits could be provided for uninsured patients. Similar efforts could facilitate the coordination of care with other providers or settings in the community for uninsured patients, as needed.

There were several limitations with this study. First, analyses were limited to the included measures capturing quality of primary care, sociodemographic characteristics, and health status. Nevertheless, this study captured a number of important indicators that are known to be associated with health care disparities, such as those describing socioeconomic status and general perceived health status. Second, the cross-sectional design of this study limited our ability to make causal inferences about the effects of race/ethnicity or insurance sta- tus on the quality of primary care experienced. Third, the self-reported nature of responses captured by the patient survey may be subject to recall or response bias. Finally, this study examined the experiences with primary care, but did not examine the links with health outcomes. Future research is needed to investigate the relationship between distinguishing aspects of primary care and health outcomes in patients at HCs.

Despite these limitations, the findings in this study lend support to the critical role that HCs serve as safety-net providers for the nation's vulnerable populations. HCs provide a broad scope of high-quality primary care services to diverse communities. Thus, HCs can be considered an effective primary care model for addressing health and health care disparities and delivering quality care to those who need it most.

\section{References}

1. Institute of Medicine. Crossing the quality chasm: the Institute of Medicine health care quality initiative. Washington, DC: National Academies Press; 2006.

2. Agency for Healthcare Research and Quality. CAHPS clinician \& group surveys. Available from: http://cahps.ahrq.gov/clinician_group/. Accessed October 19, 2012.

3. Scholle S, Vuong O, Ding L, et al. Development of and field-test results for the CAHPS PCMH survey. Med Care 2012;50(Suppl):S2-10.

4. Pascoe G. Patient satisfaction in primary health care: a literature review and analysis. Eval Program Plann 1983;6:185-210.

5. Keating NL, Green DC, Kao AC, Gazmararian JA, Wu VY, Cleary PD. How are patients' specific ambulatory care experiences related to trust, satisfaction, and considering changing physicians? J Gen Intern Med 2002;17:29-39.

6. Starfield B, Shi L, Macinko J. Contribution of primary care to health systems and health. Milbank Q 2005;83:457-502.

7. Guide to clinical preventive services: recommendations of the U.S. Preventive Services Task Force. Washington, DC: Agency for Healthcare Research and Quality; 2009.

8. Institute of Medicine. A manpower policy for primary health care. Washington, DC: National Academy of Sciences; 1978.

9. Allhoff F, Chrishon K, Jarosch J, et al. An Ethical Force Program consensus report: improving communication-improving care. Chicago, IL: American Medical Association; 2006. Available from: http:// www.ama-assn.org/ama1/pub/upload/mm/369/ef_imp_ comm.pdf. Accessed October 19, 2012.

10. Safran DG, Taira DA, Rogers WH, Kosinski M, Ware 
JE, Tarlov AR. Linking primary care performance to outcomes of care. J Fam Pract 1998;47:213-9.

11. Starfield B. Primary care: concept, evaluation, and policy. New York: Oxford University Press; 1992.

12. Robinson J, Shavers V. The role of health insurance coverage in cancer screening utilization. J Health Care Poor Underserved 2008;19:842-56.

13. Hadley J. Insurance coverage, medical care use, and short-term health changes following an unintentional injury or the onset of a chronic condition. JAMA 2007;297:1073-84.

14. Hadley J, Cunningham P. Availability of safety net providers and access to care of uninsured persons. Health Serv Res 2004;39:1527-46.

15. Office of Minority Health and Health Disparities. Eliminating racial and ethnic health disparities. Atlanta: Centers for Disease Control and Prevention; 2011.
16. Institute of Medicine. Unequal treatment: confronting racial and ethnic disparities in health care. Washington, DC: National Academies Press: 2002.

17. Taylor J. The fundamentals of community health centers. Washington, DC: The George Washington University National Health Policy Forum; 2004.

18. Health Resources and Services Administration. Primary care: the heath center program. National data. 2009 National total summary data. Available from: http:// bphc.hrsa.gov/healthcenterdatastatistics/nationaldata/ 2009/2009nattotsumdata.html. Accessed March 15, 2012.

19. Health Resources and Services Administration. Primary care: the heath center program. Authorizing legislation. Available from: http://bphc.hrsa.gov/ policiesregulations/legislation/index.html. Accessed March 15, 2012. 